\title{
CAPACIDADE MÁXIMA DE ADSORÇÃO DE FÓSFORO E CONSTANTE DE ENERGIA DE LIGAÇÃO EM LATOSSOLO BRUNO EM RAZÃO DE DIFERENTES AJUSTES DO MODELO DE LANGMUIR ${ }^{(1)}$
}

\author{
Clovisson Menotti Boeira de Oliveira ${ }^{(2)}$, Luciano Colpo Gatiboni ${ }^{(3)}$, David José \\ Miquelluti $^{(3)}$, Thomas Jot Smyth(4) \& Jaime Antonio Almeida ${ }^{(3)}$
}

\begin{abstract}
RESUMO
A capacidade máxima de adsorção de fósforo (CMAP) é um parâmetro bastante útil para caracterizar a capacidade de adsorção de fósforo $(\mathrm{P})$ do solo e, por isso, $o$ modelo de Langmuir, que possibilita essa estimativa, é bastante difundido. Porém, se o ajuste da equação for realizado por modelos não lineares ou linearizados, ou se forem escolhidos modelos de região única ou múltiplas, nem sempre os valores estimados da CMAP e da constante de energia de ligação (k) são semelhantes. $O$ objetivo deste trabalho foi avaliar o efeito do uso de diferentes métodos de ajuste do modelo de Langmuir sobre os valores estimados de CMAP e k. Para isso, utilizouse um único solo de alta capacidade de adsorção de $P$, o qual foi misturado a quantidades crescentes de areia lavada, construindo-se sistemas com capacidades de sorção crescentes, mas com a fase sólida constituída da mesma mineralogia. Foi utilizado solo do horizonte B de um Latossolo Bruno com $800 \mathrm{~g} \mathrm{~kg}^{-1}$ de argila, o qual foi misturado com areia em quantidades para obterem-se solos artificiais com 0 , $200,400,600$ e $800 \mathrm{~g} \mathrm{~kg}^{-1}$ de argila. Esses solos artificiais foram incubados por 30 dias com calcário para elevar o $\mathrm{pH}\left(\mathrm{H}_{2} \mathrm{O}\right)$ até 6,0 e, após, foram secos em estufa e peneirados. Foram realizadas as isotermas de adsorção e os dados ajustados pelo modelo de Langmuir, usando os seguintes métodos: NLin - não linear com região única; L-1R - linearização com região única; L-2RG - linearização com duas regiões, ajuste gráfico; L-3RG - linearização com três regiões, ajuste gráfico; L-2RE linerização com duas regiões, ajuste estatístico. Os resultados evidenciaram que todos os métodos utilizados estimaram valores de CMAP proporcionais ao teor de
\end{abstract}

(1) Extraído da Dissertação de Mestrado do primeiro autor. Realizado com recursos do CNPq e FAPESC. Recebido para publicação em 8 de maio de 2014 e aprovado em 19 de agosto de 2014.

(2) Doutorando no Programa de Pós-graduação em Ciência do Solo, Universidade do Estado de Santa Catarina - UDESC. Av. Luís de Camões, 2090. CEP 88520-000 Lages (SC). E-mail clovissonboeira@yahoo.com.br

(3) Professor, Departamento de Solos e Recursos Naturais, UDESC. E-mail lgatiboni@gmail.com,dmiquell@gmail.com, jaime.almeida@udesc.br

(4) Professor, Department of Soil Science, North Carolina State University. PO Box 7619. Raleigh-NC, 27695-7619, USA. E-mail: jot_smyth@ncsu.edu 
argila dos solos e poderiam ser usados para caracterizar os solos. Contudo, quando utilizados ajustes com mais de uma região de adsorção, os valores da CMAP para a última região foram sensivelmente superiores àqueles observados após a incubação do solo com doses de $\mathrm{P}$ em um teste adicional. Isso indica que a CMAP da última região deve ser evitada como caracterizadora da capacidade de adsorção do solo. Conforme era esperado, os valores de $\mathrm{k}$ foram proporcionais aos teores de argila do solo na primeira (ou única) região dos modelos linearizados; contudo, não seguiram essa tendência no modelo não linear, recomendando-se cautela na interpretação da constante $k$ ajustada por modelos não lineares.

Termos de indexação: isotermas, Pmax, adsorção máxima, modelos de adsorção, sorção de fósforo.

\title{
SUMMARY: MAXIMUM PHOSPHORUS ADSORPTION CAPACITY AND BINDING ENERGY CONSTANT OF AN OXISOL FITTING DIFFERENT LANGMUIR MODELS
}

\begin{abstract}
Maximum phosphorus adsorption capacity (Pmax) is a useful tool for characterizing the phosphorus $(P)$ sorption of soils, and the Langmuir model, which allows this estimate, has widespread use. However, estimated values of Pmax and the binding energy constant ( $k$ ) may be quite different when fitted to non-linear or linearized Langmuir models, or models with single or multiple regions. The objective of this study was to compare the effect of using different methods to fit the Langmuir model to the estimation of Pmax and $k$ values. A single soil with high Pmax was mixed with increasing amounts of sand, producing artificial soils with increasing $P$ adsorption capacities but with the same clay mineralogy. Soil from the $B$ horizon of an Oxisol containing $800 \mathrm{~g} \mathrm{~kg}^{-1}$ clay was mixed with washed sand in amounts necessary to obtain samples with 0, 200, 400,600, and $800 \mathrm{~g} \mathrm{~kg}^{-1}$ of clay. These artificial soils were incubated for 30 days with lime to raise the $\mathrm{pH}$ in water level to 6.0. The adsorption isotherms were constructed and the data fitted using the following Langmuir model approaches: NLin - nonlinear with a single region; $L-1 R$ - linearization with a single region; $L-2 R G$ - linearization with two regions and graphical adjustment; $L-3 R G$ - linearization with three regions and graphical adjustment; and L-2RE - linearization with two regions and statistical fitting. The results showed that the Pmax values estimated by all methods were dependent on the clay content of soils and could be used to characterize the Padsorption of the soil. However, when fitted to more than one adsorption region, the Pmax values for the last region were significantly higher than those observed after incubation of the soil with different P levels. Therefore, the Pmax of the last region in multiregion models may not be appropriate for characterizing soil P adsorption capacity. As expected, the $k$ values were dependent on soil clay content in the first (or single) region of the linearized models, but they did not follow this trend in the nonlinear model. Thus, caution should be taken in interpretation of the $k$ constant fitted to nonlinear models.
\end{abstract}

Index terms: isotherms, Pmax, maximum adsorption, adsorption models, phosphorus sorption.

\section{INTRODUÇÃO}

No processo de adsorção, o nutriente adicionado na solução é adsorvido pela fase sólida, sendo a velocidade de adsorção e a quantidade total adsorvida dependentes das características químicas e mineralógicas do material sorvente e das características químicas do adsorbato. A adsorção e dessorção de $\mathrm{P}$ do solo consideram dois compartimentos interligados, a solução do solo e a fase sólida, que armazena o nutriente que abastece a primeira. A cinética de como o primeiro compartimento é abastecido pelo segundo, chamado fator capacidade, representa o poder tampão de $\mathrm{P}$, o qual é controlado, entre outras coisas, pela energia de ligação do nutriente na fase sólida (Barrow, 2008). Como o P absorvido pelas plantas é o que está na solução do solo, a disponibilidade de $\mathrm{P}$ para as culturas é constituida pelas formas de $\mathrm{P}$ na fase sólida que potencialmente pode repor a solução do solo. Contudo, à medida que aumenta a capacidade tampão de $\mathrm{P}$ do solo, a quantidade necessária para manter a mesma concentração de P na solução é maior, acarretando em maiores doses de fertilizantes a serem aplicadas no solo. Como a CMAP informa o tamanho do complexo sortivo do solo, é um parâmetro bastante utilizado para caracterização de solos e consequências de manejo que influenciam a dinâmica de P no solo (Novais \& Smyth, 1999).

A adsorção de $\mathrm{P}$ aos coloides inorgânicos do solo (argilas silicatadas e oxi-hidróxidos) ocorre com diferentes energias de ligação, dependendo do tipo de 
sítio adsorvente (Parfitt, 1978) e do grau de sua saturação com P (Rheinheimer et al., 2003; Barrow, 2008). Durante o processo de adsorção de P, ocorrem três estádios temporais: no estádio 1, predominam a neutralização das cargas positivas (grupos $\mathrm{R}-\mathrm{OH}_{2}^{+}$); no estádio 2 , ocorrem as trocas de ligantes; e, no estádio 3 , há interações de caráter eletrostático entre o $\mathrm{PO}_{4} \mathrm{e}$ a superfície dos coloides (Muljadi et al., 1966a,b; Parfitt, 1978). Esses estádios estão relacionados com a afinidade do $\mathrm{P}$ com os sítios de adsorção em cada fase da adsorção e, consequentemente, com distintas energias de ligação (Muljadi et al., 1966c). Como pressupostos do mecanismo de adsorção, esses autores enumeram que os sítios de adsorção devem ser isolados e independentes quanto à energia de retenção e que as moléculas adsorvidas não devem interagir umas com as outras. Dessa maneira, a adsorção de P ao solo é complexa e envolve fases distintas, que, dependendo do grau de saturação do solo com $\mathrm{P}$, fazem com que a quantidade e o tipo de sítios adsorventes variem, alterando, portanto a energia de ligação a esses sítios e a possibilidade de dessorção do P.

Modelos matemáticos são utilizados para descrever as proporções de $\mathrm{P}$ adsorvido à fase sólida em razão da adição de quantidades crescentes de P na solução ou variação do tempo de contato do $\mathrm{P}$ com o solo (Barrow, 2008); entretanto, alguns autores postulam que a descrição do fenômeno de adsorção não é devidamente predita por modelos de região única, já que esses tratam, de maneira igual, diferentes fases do processo de adsorção. O modelo matemático mais difundido é o de Langmuir, que passou a ser utilizado em estudos da adsorção de P porque possibilita obtenção da CMAP do solo e da constante "k", relacionada à energia de ligação desse elemento ao solo (Novais \& Smyth, 1999). Embora bastante utilizado, segundo esses autores, o modelo de Langmuir apresenta alguns problemas por causa das seguintes pressuposições: a energia de adsorção é constante; a adsorção é vetorial, não ocorrendo interações laterais no plano da superfície; e a máxima adsorção possível corresponde a uma camada monomolecular na superfície do coloide. Para um sistema complexo e heterogêneo como o solosolução, esses postulados não são atendidos, pois os sítios de adsorção não são uniformes; podem ocorrer interações não vetoriais e, ainda, o $\mathrm{PO}_{4} \mathrm{em}$ altas concentrações pode precipitar com outros íons na solução. Por isso, Barrow (2008) considerou o modelo de Langmuir inadequado para estudos de adsorção de íons em solos, recomendando o modelo de Freundlich para esse fim. Além disso, um único valor de energia de ligação fornecido pelo modelo de Langmuir de região única também não parece adequado ao se levar em conta as três fases de adsorção descritas por Muljadi et al. (1966a,b) e Parfitt (1978).

Embora o modelo não linear de região única de Langmuir seja amplamente utilizado, para Bolster \& Hornberge (2007), esse ajuste subestimou o P sorvido; porém, os ajustes foram significativamente superiores quando usaram modelos linearizados de Langmuir.
Contudo, Harter (1984), Bolster \& Hornberger (2007) e Barrow (2008) alertaram que a linearização dos dados causa dificuldades estatísticas, pois se perde a independência entre as variáveis, diminuindo artificialmente o erro e aumentando o ajuste do modelo aos dados. Já sobre o uso de modelo com múltiplas regiões, Wang \& Li (2010) afirmaram que a utilização da isoterma de Langmuir com duas regiões apresentou bom ajuste na avaliação do potencial de retenção de $\mathrm{P}$ por sedimentos de uma bacia hidrográfica. O uso dessas equações com múltiplas regiões de adsorção tem sido por vezes adotados, e a justificativa pode ser tanto teórica, com base nas diferentes fases de adsorção do $\mathrm{P}$ ao solo, como também prática, em razão de o modelo de múltiplas regiões apresentar melhor ajuste dos segmentos da equação aos dados (Hussain et al., 2006).

Com o uso de modelos de múltiplas regiões, são encontrados mais de um valor de $\mathrm{k}$, que representam a energia de ligação nas diferentes fases de adsorção, além de um valor de CMAP para cada fase. Nesse caso, para caracterizar o poder sortivo do solo, pode-se escolher a CMAP da última região, que representa a máxima adsorção após todas as fases de adsorção ou, ainda, escolher a CMAP de uma das regiões como a representante do solo, como proposto por Alvarez V. \& Fonseca (1990). Segundo esses autores, em um modelo com três regiões, a região 2 é a que melhor cumpre as pressuposições do modelo de Langmuir em estudos com solos. Farias et al. (2009) verificaram que utilizando a região 2 para cálculos da CMAP, quando comparada com o método da regressão não linear, a linearização da segunda região de adsorção subestimou em $33 \%$, em média, os valores de CMAP nos solos mais intemperizados e em $31 \%$, nos menos intemperizados. No entanto, Corrêa et al. (2011) utilizaram a região 2 da isoterma de Langmuir linearizada e encontraram elevados coeficientes de correlação da CMAP, indicando que o modelo de adsorção foi capaz de estimar a adsorção de $\mathrm{P}$ nos solos analisados.

Pelo exposto, há várias maneiras para ajustar o modelo de Langmuir aos dados de adsorção de $\mathrm{P}$ do solo, porém sem consenso sobre qual a mais adequada. A hipótese é que, usando amostras com diferentes teores de argila, mas com exatamente a mesma composição mineralógica, possa-se avaliar com maior clareza as consequências da escolha de diferentes ajustes do modelo de Langmuir sobre as constantes CMAP e k. Assim, o objetivo deste trabalho foi avaliar a precisão de estimativa da CMAP e k por diferentes ajustes do modelo de Langmuir, usando amostras de um solo misturado com diferentes proporções de areia.

\section{MATERIAL E MÉTODOS}

O experimento foi conduzido no Centro de Ciências Agroveterinárias (CAV) da Universidade do Estado de 
Santa Catarina (UDESC), em Lages, SC. O solo utilizado foi Latossolo Bruno, coletado do horizonte subsuperficial (Bw - 140/220 cm) com alto teor de argila $\left(800 \mathrm{~g} \mathrm{~kg}^{-1}\right)$, em área de campo nativo. A mineralogia da fração argila do solo utilizado está caracterizada em Almeida et al. (2003) e é composta predominantemente por caulinita, seguida de goethita, hematita e argilas $2: 1$ com hidróxi-Al entrecamadas. Após a coleta, o solo foi seco em estufa a $60^{\circ} \mathrm{C}$ e passado em peneira com malha de $2 \mathrm{~mm}$. Para o experimento, construíram-se unidades experimentais com diferentes teores de argila, ns quais o solo foi misturado com diferentes quantidades de areia peneirada (com tamanho de partícula entre 0,5 e 1,0 mm), lavada previamente com $\mathrm{HCl} 0,1 \mathrm{~mol} \mathrm{~L}^{-1}$ e água destilada. Foram feitas misturas de areia e solo nas proporções de $100 \%$ de areia $+0 \%$ solo; $75 \%$ de areia $+25 \%$ solo; $50 \%$ areia $+50 \%$ solo; $25 \%$ areia $+75 \%$ solo; $0 \%$ areia $+100 \%$ solo, constituíndo as unidades experimentais com 0, 200, 400, 600 e $800 \mathrm{~g} \mathrm{~kg}^{-1} \mathrm{de}$ argila, respectivamente.

As unidades experimentais foram acondicionadas em baldes plásticos com tampa, colocadas em casa de vegetação e incubadas por 30 dias a $80 \%$ da capacidade de campo para estabilização de propriedades físicoquímicas. $\mathrm{O}$ teor de umidade na capacidade de campo das amostras foi estimado após saturação e $24 \mathrm{~h}$ de drenagem, seguindo o método descrito em Casaroli \& van Lier (2008). Após a incubação, as amostras foram secas em estufa e passadas em peneira com malha de $2 \mathrm{~mm}$ e reincubadas por 30 dias com calcário filler $(\mathrm{PRNT}=98,1 \%)$ para correção do $\mathrm{pH}\left(\mathrm{H}_{2} \mathrm{O}\right)$ até 6,0 , sendo as doses calculadas pelo índice SMP, conforme recomendações da Comissão de Química e Fertilidade do Solo local (CQFSRS/SC, 2004). Após a incubação, as unidades experimentais apresentaram as caraterísticas físicas e químicas apresentadas no quadro 1.

Ao final das incubações, as amostras das unidades experimentais, passadas em peneira de $2 \mathrm{~mm}$, foram submetidas aos seguintes procedimentos para confecção de isotermas: pesou-se, em tubo de centrífuga, 0,5 g de solo seco em estufa, em quadruplicata, ao qual foram aplicados $15 \mathrm{~mL}$ de soluções de $\mathrm{P}$ preparadas em solução de fundo de $\mathrm{CaCl}_{2}$ $0,01 \mathrm{~mol} \mathrm{~L}^{-1}$, que corresponderam às doses de 0,30 , 60, 120, 180, 240, 480, 960, 1920, 2880, $3840 \mathrm{mg} \mathrm{kg}^{-1}$ de $\mathrm{P}$ solo. Utilizou-se como fonte de $\mathrm{P}_{\mathrm{KH}_{2}} \mathrm{PO}_{4}$ p.a.. As amostras foram agitadas por $16 \mathrm{~h}$ em agitador tipo end-over-end a $30 \mathrm{rpm}$. Após a agitação, separou-se o solo da solução por meio da centrifugação a $5.000 \mathrm{rpm}$ por 14 min, procedendo-se às determinações de $\mathrm{P}$ no sobrenadante colorimetricamente após a redução do complexo fosfomolíbdico por ácido ascórbico, conforme método de Murphy \& Riley (1962).

Para cada teor de argila, foram ajustadas equações de Langmuir por cinco diferentes métodos: NLin - não linear com região única; L-1R - linearização com região única; L-2RG - linearização com duas regiões, ajuste gráfico ou visual; L-3RG - linearização com três regiões, ajuste gráfico; e L-2RE - linerização com duas regiões, ajuste estatístico. Para o ajuste não linear com região única, os dados foram submetidos ao procedimento proc nlin do pacote estatístico SAS, versão 9.1 (SAS, 2003), ajustando-se o modelo de Langmuir: $\mathrm{q}=(\mathrm{k} \times \mathrm{CMAP} \times \mathrm{C}) /(1+\mathrm{k} \times \mathrm{C})$, em que $\mathrm{q}=\mathrm{P}$ sorvido, $\mathrm{k}=$ constante relacionada à energia de ligação, $\mathrm{CMAP}=$ capacidade de adsorção de $\mathrm{P}$ e C $=\mathrm{P}$ na solução de equilíbrio. A linearização dos dados e o ajuste pelos modelos com uma, duas e três regiões foram realizados utilizando a relação $\mathrm{C} / \mathrm{q}=\mathrm{f}(\mathrm{C})$, em que $\mathrm{C}$ é a concentração de $\mathrm{P}$ na solução de equilíbrio $\left(\mathrm{mg} \mathrm{L}^{-1}\right)$; e q, o $\mathrm{P}$ adsorvido $\left(\mathrm{mg} \mathrm{g}^{-1}\right)$. Após a linearização, a obtenção dos parâmetros k e CMAP foi calculada, conforme descrito em Novais \& Smyth (1999).

Para identificar as regiões de adsorção pelo método do ajuste gráfico nos métodos L-2RG e L-3RG, usouse o método proposto por Muljadi et al. (1966a,b), em que a separação de cada região de adsorção foi determinada visualmente a partir dos dados plotados em gráficos, ajustando uma equação linear para cada uma das regiões.

No ajuste L-2RE, foi aplicado um método estatístico para identificar as regiões de adsorção de $\mathrm{P}$ ajustando as isotermas de Langmuir por meio de análise de

Quadro 1. Características físicas e químicas das unidades experimentais, após incubação por 30 dias com água e mais 30 dias com o corretivo de acidez

\begin{tabular}{|c|c|c|c|c|c|c|c|c|c|c|c|}
\hline Tratamento & Argila $^{(1)}$ & Silte & Areia & $\mathrm{pH}\left(\mathrm{H}_{2} \mathrm{O}\right)^{(2)}$ & $\mathbf{P}$ & $\mathbf{K}$ & $\mathbf{C a}$ & Mg & Al & $\mathrm{H}+\mathrm{Al}$ & MO \\
\hline & 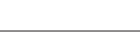 & $\mathrm{g} \mathrm{kg}^{-1}$ & 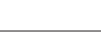 & & \multicolumn{2}{|c|}{$\mathrm{mg} \mathrm{kg}^{-1}$} & \multicolumn{4}{|c|}{$\mathrm{cmol}_{\mathrm{c}} \mathrm{dm}^{-3}$} & $\mathrm{~g} \mathrm{~kg}^{-}$ \\
\hline 0 & 0 & 0 & 1000 & 5,7 & 1,8 & 7 & 0,0 & 0,0 & 0,4 & 0,7 & nd \\
\hline 200 & 200 & 50 & 750 & 5,4 & 1,7 & 8 & 0,1 & 0,1 & 0,2 & 1,7 & nd \\
\hline 400 & 400 & 90 & 510 & 5,3 & 1,9 & 7 & 0,8 & 0,5 & 0,1 & 2,0 & nd \\
\hline 600 & 600 & 130 & 270 & 5,8 & 1,8 & 9 & 2,5 & 1,4 & 0,0 & 2,0 & 2,0 \\
\hline 800 & 800 & 180 & 20 & 5,7 & 1,6 & 10 & 3,5 & 2,1 & 0,0 & 2,2 & 3,0 \\
\hline
\end{tabular}

(1) Caracterização granulométrica determinada pelo método da pipeta (Embrapa, 1997). (2) Caracterização química de acordo com métodos descritos em Tedesco et al. (1995), sendo $\mathrm{pH}$ na relação 1:1; H+Al avaliado por potenciometria, após adição de solução de SMP; P e K extraídos por Mehlich-1; Ca, Mg e Al trocáveis extraídos por $\mathrm{KCl}$ 1,0 mol L'-1; e MO: matéria orgânica, oxidada por solução sulfocrômica. nd: não detectado. 
regressão. Para a estimação dos parâmetros por esse procedimento, foi ajustado um modelo de regressão segmentada, com dois segmentos, utilizando-se o método de mínimos quadrados, e a determinação do ponto ótimo de separação entre os dois segmentos foi efetuada pela maximização do coeficiente de determinação $\left(\mathrm{r}^{2}\right)$. Essas análises foram realizadas com o auxílio do software R ( $R$ Development Core Team, 2012).

Como todos os métodos de ajuste de Langmuir descritos anteriormente estimaram a CMAP, houve necessidade de execução de um teste complementar de adsorção via incubação do solo para definir qual das CMAP calculadas seria a mais indicada para uso. Esse teste foi realizado da seguinte maneira: as unidades experimentais com 0,200, 400, 600 e 800 $\mathrm{g} \mathrm{kg}^{-1}$ de argila foram incubadas por 20 dias com umidade na capacidade de campo e com sete doses de $\mathrm{P}$ variando desde zero até próximo da CMAP dos solos, com três repetições. Após a incubação, foi determinado o P solúvel pelo seguinte procedimento: $1,0 \mathrm{~g}$ de solo (base seca) foi colocado em tubos de centrífuga, adicionando $10 \mathrm{~mL}$ de água destilada e agitando por 60 min em end-over-end. Após centrifugação, o teor de P no extrato foi determinado pelo método de Murphy \& Riley (1962). Aos dados de $\mathrm{P}$ adicionado versus $\mathrm{P}$ na solução, as CMAP foram ajustadas por regressões segmentadas usando o mesmo procedimento descrito anteriormente para L-2RE, cujo ponto de intersecção entre as duas regressões foi considerado a capacidade real de adsorção de $\mathrm{P}$ do solo.

\section{RESULTADOS E DISCUSSÃO}

$\mathrm{Na}$ figura 1a, são apresentadas as curvas de adsorção de $\mathrm{P}$ dos solos e o ajuste não linear pelo modelo de Langmuir (NLin), em que se pode observar que a adsorção de $\mathrm{P}$ foi maior em unidades experimentais com textura mais argilosa, conforme era esperado. A modelagem não linear das curvas apresentou alguns desvios em relação aos dados observados, o que resultou em coeficientes de determinação da ordem de 0,88 a 0,98 (Quadro 2). Os valores de CMAP estimados pelo modelo NLin foram de 33, 354, 1001, 1630 e $2111 \mathrm{mg} \mathrm{kg}^{-1}$ de $\mathrm{P}$ nas respectivas unidades com 0, 200 , 400, 600 e $800 \mathrm{~g} \mathrm{~kg}^{-1}$ de argila (Quadro 2). O valor da CMAP foi proporcional ao teor de argila, comportamento semelhante ao observado em diversos trabalhos (Valladares et al., 2003; Falcão \& Silva, 2004; Ranno et al., 2007; Corrêa et al., 2011).

Pelos coeficientes de determinação (Quadro 2) e pela figura 1a, fica evidente que o traçado da equação não linear de Langmuir não evidencia bom ajuste em relação aos pontos observados e isso é uma desvantagem desse modelo, o qual, segundo Barrow (2008), raramente reproduz a correta forma de curvas de sorção, principalmente pelas restrições matemáticas do modelo; entre essas, a necessidade de passar pela origem e de ter que se chegar a um valor de máxima adsorção.

Adicionalmente, outra inconsistência foi observada com o ajuste NLin, referindo-se à constante relacionada com a energia de ligação, k (Quadro 2). Nesse experimento, o solo original é o mesmo para todos os tratamentos e apenas foi misturado com quantidades crescentes de areia. Assim, há certeza de que a mineralogia da fração argila é idêntica em todos os solos (caulinita, seguida de óxidos de Fe), variando apenas a sua quantidade em cada tratamento. Com isso, a afinidade por $\mathrm{P}$, medida pela constante $\mathrm{k}$, deveria ser também proporcional ao teor de argila dos tratamentos; porém, isso não foi observado para o ajuste não linear (Figura 2). Houve variação de 0,62 a 7,95 no valor dessa constante e isso ocorreu sem correlação com o teor de argila da amostra; o menor valor foi encontrado no solo com $600 \mathrm{~g} \mathrm{~kg}^{-1}$ de argila e o maior naquele com $200 \mathrm{~g} \mathrm{~kg}^{-1}$ (Figura 2a, Quadro 2). Isso indica que o parâmetro $\mathrm{k}$ pode variar durante o processo de ajuste não linear do modelo a fim de melhorar a aderência do modelo aos dados observados. Por isso, a interpretação dos resultados da variável $\mathrm{k}$ deve ser feita com cuidado em ajustes NLin, pois pode não ser suficientemente precisa para ser utilizada.

Quando utilizada a linearização dos dados e o ajuste pelo modelo de adsorção de Langmuir em região única (L-1R), a CMAP variou de 24 a $2372 \mathrm{mg} \mathrm{kg}^{-1}$ para as respectivas unidades experimentais de 0 a $800 \mathrm{~g} \mathrm{~kg}^{-1}$ de argila (Quadro 2), apresentando também a proporcionalidade entre o teor de argila das amostras e a CMAP estimadas, semelhantemente ao ocorrido para o ajuste NLin. Entretanto, contrariamente ao ocorrido para o modelo NLin, a linearização trouxe coerência para a constante de energia de ligação, pois os valores calculados de $\mathrm{k}$ foram crescentes com o teor de argila e apresentaram alta correlação com eles (Figura 2a). Os coeficientes de determinação das equações L-1R foram elevados $(0,99)$, exceto para o tratamento $0 \mathrm{~g} \mathrm{~kg}^{-1}$ (Quadro 2), evidenciando que o artifício matemático da linearização reduz artificialmente o erro, como alertado por Bolster \& Hornberger (2007).

Quando a reta foi segmentada adotando o método da separação gráfica em duas (L-2RG) ou três regiões (L-3RG) pelo procedimento descrito em Muljadi et al. (1966a,b), foram observados valores de CMAP crescentes com o aumento do teor de argila dos tratamentos em todos os segmentos da equação (Quadro 2). Uma questão importante que deve ser alertada quando do ajuste de equações com duas ou mais regiões é que a CMAP calculada para a segunda e terceira regiões representa a soma das CMAPs da região em questão e as das regiões anteriores. Isso ocorre porque nas equações multirregiões a CMAP de cada região estima a quantidade de $\mathrm{P}$ que poderia ser adsorvido ao solo se fosse mantida aquela taxa de adsorção. Assim, a CMAP da região I é a quantidade 

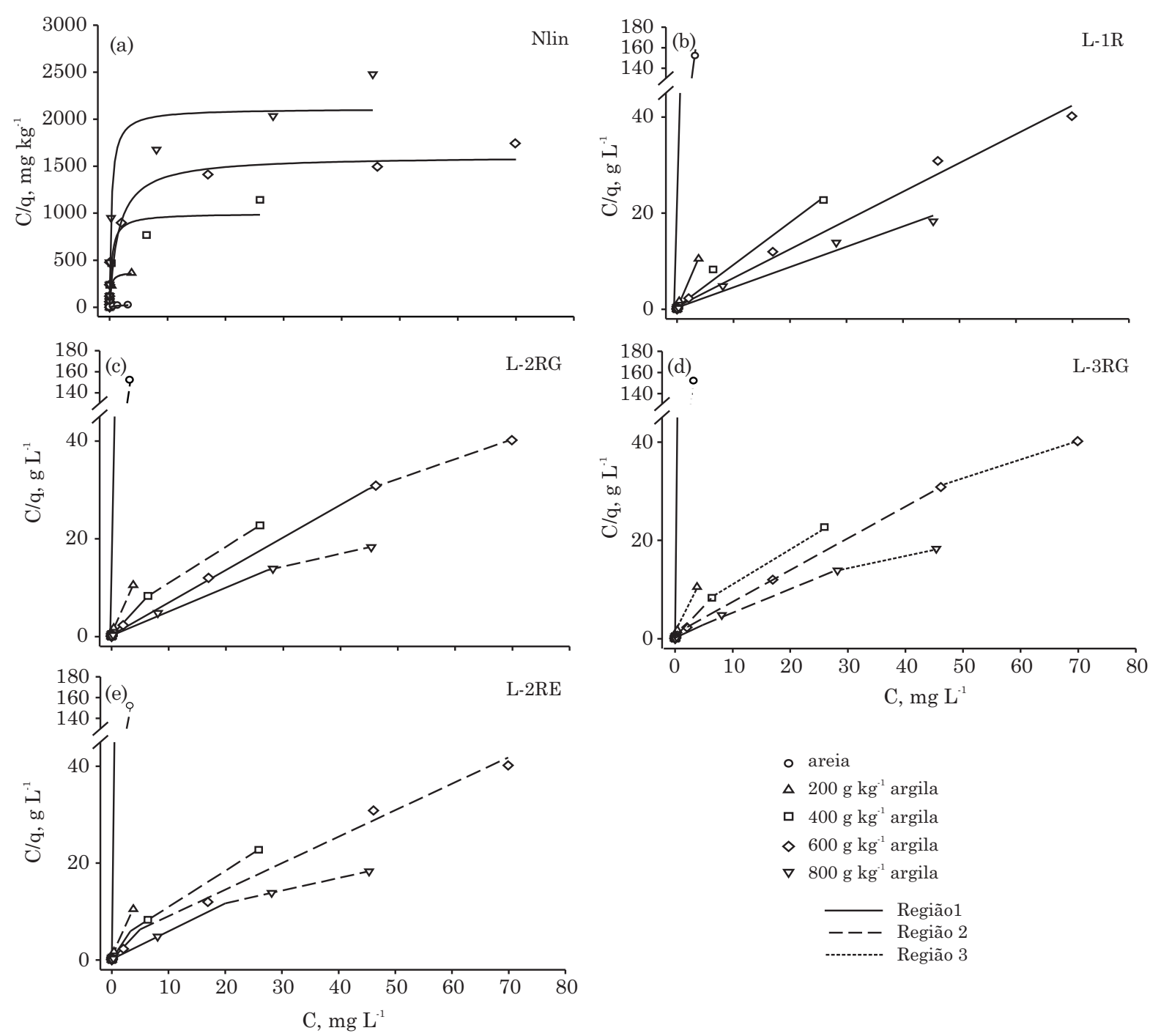

Figura 1. Isotermas de adsorção de fósforo em um solo misturado com areia em diferentes proporções para formar texturas de 0 a $800 \mathrm{~g} \mathrm{~kg}^{-1}$ de argila. Modos de ajuste do modelo: NLin = não linear com região única (a); L-1R = linearização com região única (b); L-2RG = linearização com duas regiões, ajuste gráfico (c); L-3RG = linearização com três regiões, ajuste gráfico (d); e L-2RE = linerização com duas regiões, ajuste estatístico (e).

máxima de $\mathrm{P}$ que poderia ser adsorvida ao solo naquela taxa; contudo, antes de se atingir essa CMAP, inicia a adsorção seguindo a taxa mais lenta da região II. Assim, a CMAP da região II indicaria o teor máximo de $\mathrm{P}$ adsorvido ao final da adsorção nessa nova taxa, incluindo a quantidade de $\mathrm{P}$ adsorvida na região anterior. O mesmo vale para a região III, cujo valor de CMAP inclui o P adsorvido nas regiões I e II. Dessa maneira, a CMAP é cumulativa nos modelos de multirregiões, e no quadro 2, além da CMAP, foi calculada a quantidade de $\mathrm{P}$ adsorvida em cada uma das regiões de adsorção.

Para o ajuste L-2RG, a região I explicou, em média, a adsorção de $57 \%$ do P. Já para o L-3RG, as regiões I, II e III explicaram em média 31, 29 e $40 \%$ do P adsorvido, respectivamente. No ajuste L-2RE, a região I foi responsável, em média, pela adsorção de $47 \%$ do
P. Isso indica que, independente do tipo de ajuste utilizado (L-2RG, L-3RG, L-2RE), pelo menos $30 \%$ da quantidade de $\mathrm{P}$ medida pela CMAP foram adsorvidos com alta energia de ligação ao solo, mantendo, por consequência, baixos teores de $\mathrm{P}$ na solução de equilíbrio. Independentemente do modelo de regiões múltiplas utilizado, a quantidade de $\mathrm{P}$ adsorvido em cada região foi crescente com o aumento do teor de argila do solo, exceto para a região II do ajuste L-3RG no solo com $800 \mathrm{~g} \mathrm{~kg}^{-1}$ de argila, cuja quantidade de $\mathrm{P}$ adsorvida foi menor que no solo com $600 \mathrm{~g} \mathrm{~kg}^{-1} \mathrm{de}$ argila (Quadro 2).

Com a separação das isotermas em duas ou três regiões, de maneira geral, os valores das constantes de energia de ligação (k) decresceram da região 1 até a 3, apresentando coerência na diminuição de energia de ligação com o aumento do grau de saturação de $\mathrm{P}$ 
Quadro 2. Equações para diferentes métodos de ajuste das isotermas de adsorção de $P$ pelo modelo de Langmuir em um solo misturado com areia em diferentes proporções para formar texturas de 0 a $800 \mathrm{~g} \mathrm{~kg}^{-1} \mathrm{de}_{\text {argila }}$

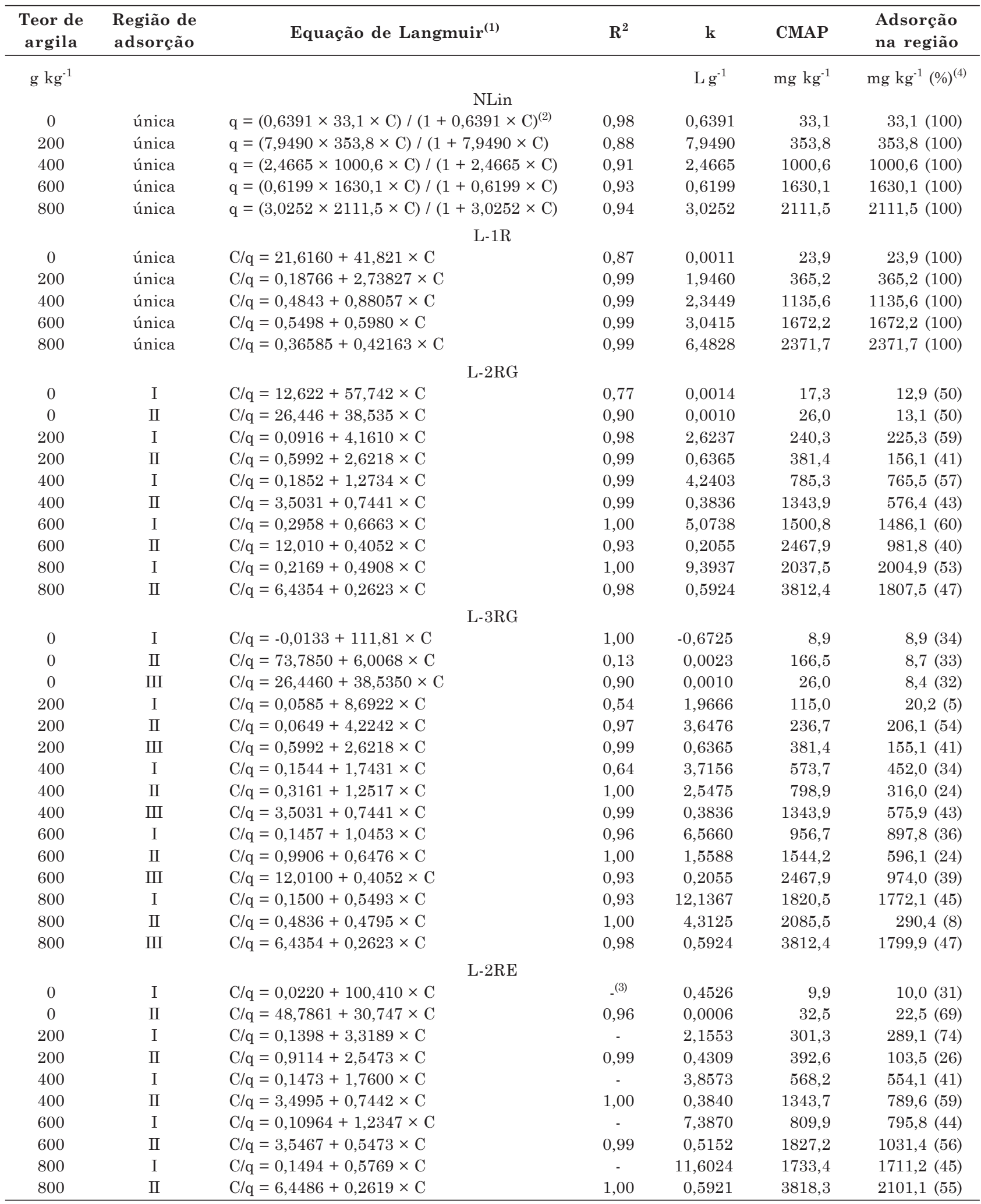

(1) Modos de ajuste do modelo: NLin = não linear com região única; L-1R = linearização com região única; L-2RG = linearização com duas regiões, ajuste gráfico; L-3RG = linearização com três regiões, ajuste gráfico; L-2RE = linerização com duas regiões, ajuste estatístico. ${ }^{(2)} \mathrm{C}$ : concentração de $\mathrm{P}$ na solução de equilíbrio; $\mathrm{q}=\mathrm{P}$ adsorvido ao solo. ${ }^{(3)} \mathrm{O}$ modelo calcula um único $\mathrm{R}^{2}$ para as duas porções da equação ajustada. ${ }^{(4)} \mathrm{O}$ valor entre parênteses indica a porcentagem de fósforo adsorvida na região, tomando como valor de 100 \% a CMAP da última região ajustada pelo modelo. 


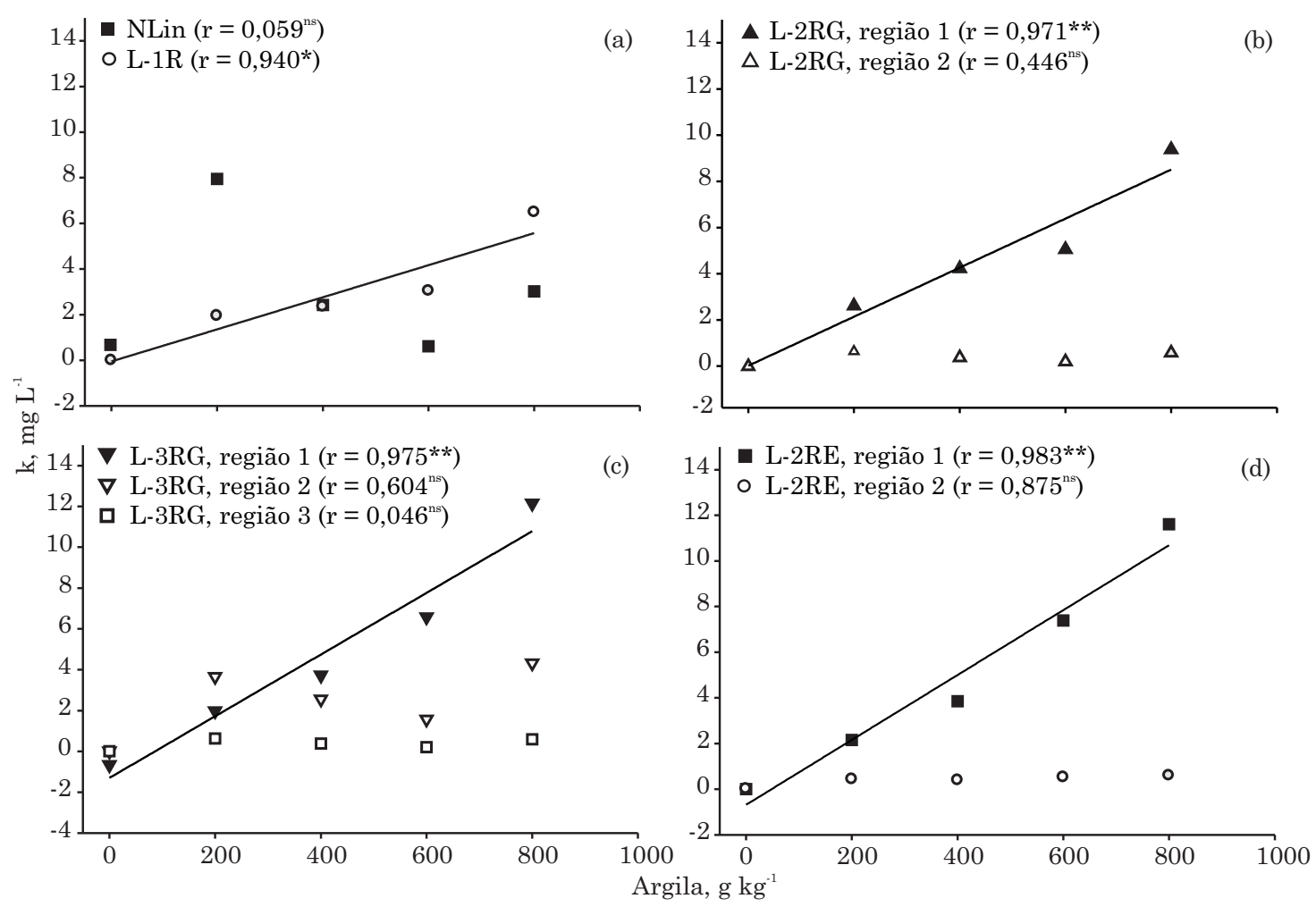

Figura 2. Relação entre o teor de argila do solo e a constante relacionada com a energia de ligação do modelo de Langmuir (k), em solos com diferentes teores de argila. Modos de ajuste do modelo: NLin = não linear com região única e L-1R = linearização com região única (a); L-2RG = linearização com duas regiões, ajuste gráfico (b); L-3RG = linearização com três regiões, ajuste gráfico (c); e L-2RE = linerização com duas regiões, ajuste estatístico (d). *: p<0,05; **: p<0,01; e ns: p>0,05.

no solo. Também, como o solo contém mineralogia composta de caulinita, óxidos de Fe e argilas 2:1 com hidroxi-Al entrecamadas, especulou-se que a diminuição nos valores de $\mathrm{k}$ se relacionam também com a gradativa saturação dos argilominerias mais ávidos por $\mathrm{P}$ com o aumento da dose adicionada, como observado por Hussain et al. (2006). Contudo, os valores de k apresentaram correlação positiva e significativa com o teor de argila apenas para a região 1 pelos ajustes L-2RG e L-3RG (Figura 2b,c). Nas demais regiões, os valores foram pouco variáveis com o teor de argila das unidades experimentais. Isso pode indicar que na região 1 ocorrem as principais reações de quimiossorção e sua energia de ligação é proporcional ao tamanho do complexo sortivo. A partir da segunda fase de adsorção, os sítios mais ávidos por $\mathrm{P}$ já foram saturados e a adsorção, que se dará principalmente por troca de ligantes, passa a ocorrer com intensidade semelhante em todos os solos, independentemente do tamanho do complexo sortivo.

Pode ser constatado na figura $1(\mathrm{~b}, \mathrm{c}, \mathrm{d}, \mathrm{e})$ que as observações registradas seguem um padrão linear em cada teor de argila e, por isso, todos os métodos de linearização (L-1R, L-2RG, L-3RG e L-2RE) apresentaram equações com elevados coeficientes de determinação. Com isso, o método de separação estatístico (L-2RE) evidenciou-se semelhante aos métodos gráficos em termos de valores estimados de CMAP e k. Durante o processo de ajuste, tentou-se, adicionalmente, fazer um ajuste estatístico dos dados com três regiões, porém não houve incremento do coeficiente de determinação do modelo com essa abordagem e, por isso, esses dados não foram apresentados.

Neste trabalho, o uso de um único tipo de solo misturado com diferentes quantidades de areia permitiu hipotetizar que o método de ajuste da equação de Langmuir mais adequado seria aquele em que a CMAP fosse proporcional ao teor de argila da amostra. Essa relação é demonstrada na figura 3, em que pode ser observado que tanto no modelo não linear quanto nos linearizados, essa proporcionalidade foi atingida. Assim, por essa hipótese, todos os métodos seriam adequados para estimar a CMAP. Contudo, quando se utilizam múltiplas regiões na isoterma, como essas são acumulativas, conforme discutido anteriormente, chegam-se a valores maiores de CMAP na última região do que aqueles ajustados para uma única região; isso gera a dúvida de qual das CMAP deve ser usada como a capacidade de sorção de $\mathrm{P}$ do solo. Alvarez V. \& Fonseca (1990) recomendaram que, quando se usa a isoterma de Langmuir com três regiões, seria mais coerente usar a CMAP da região 2 como referencial do solo, uma vez que na região 3 tomam parte as 
reações em longo prazo e, ou, precipitação de compostos fosfatados.

Embora todos os métodos de ajuste utilizados neste trabalho tenham coerentemente chegado a valores de CMAP proporcionais aos teores de argila, os valores absolutos são muito divergentes e, assim, permanece a dúvida de qual CMAP seria a mais adequada para caracterizar o poder de sorção do solo. Para tentar identificar qual dos valores seria mais adequado, foi realizado um estudo complementar em que os solos com diferentes teores de argila foram incubados com doses de $\mathrm{P}$ por 20 dias, e após foi medido o teor de $\mathrm{P}$ solúvel em água, ou fator intensidade. Nesse procedimento, partiu-se da premissa que à medida que a adição de $\mathrm{P}$ se aproxima da capacidade máxima de adsorção de $\mathrm{P}$ do solo, a maior parte do $\mathrm{P}$ adicionado passa a ficar na solução do solo e, com isso, o ponto de intersecção entre as duas regressões ajustadas representaria a capacidade real de adsorção de $\mathrm{P}$ do solo.

Os resultados do ensaio complementar apresentaram que para o tratamento sem argila $\left(0 \mathrm{~g} \mathrm{~kg}^{-1}\right.$ de argila) praticamente todo o $\mathrm{P}$ adicionado foi detectado na água, evidenciando a capacidade de adsorção praticamente nula da fase sólida desse tratamento (dados não apresentados). Já para os tratamentos de 200 a $800 \mathrm{~g} \mathrm{~kg}^{-1}$ de argila, demonstrados na figura 4 , o ajuste de uma regressão segmentada com duas regiões indicou que a partir de valores adicionados de 367, 712, 1.080 e $1.384 \mathrm{mg} \mathrm{kg}^{-1}$ de $\mathrm{P}$ passou a ocorrer mais incremento de $\mathrm{P}$ na solução por unidade adicionada do que adsorção na fase sólida, para os tratamentos 200, 400, 600 e $800 \mathrm{~g} \mathrm{~kg}^{-1}$ de argila, respectivamente. Esses valores representariam uma aproximação da real capacidade de adsorção do solo com 20 dias de contato do P com o solo. Ao se comparar esses resultados com as CMAPs estimadas pela isoterma de Langmuir, observou-se que ao optar pelos ajustes com linearização e regiões múltiplas, não se deve utilizar como referência a CMAP da última região, pois os valores são sensivelmente maiores que os estimados após uma incubação de 20 dias do solo com doses de P.

Foram ajustadas equações entre o valor de convergência das equações segmentadas da figura 4 e as diferentes CMAPs calculadas (dados não apresentados). Todas as equações foram lineares, apresentaram alto coeficiente de determinação, e os coeficientes angulares calculados foram: 1,72 (NLin); 1,91 (L-1R); 1,79 (L-2RG, região I); 3,33 (L-2RG, região

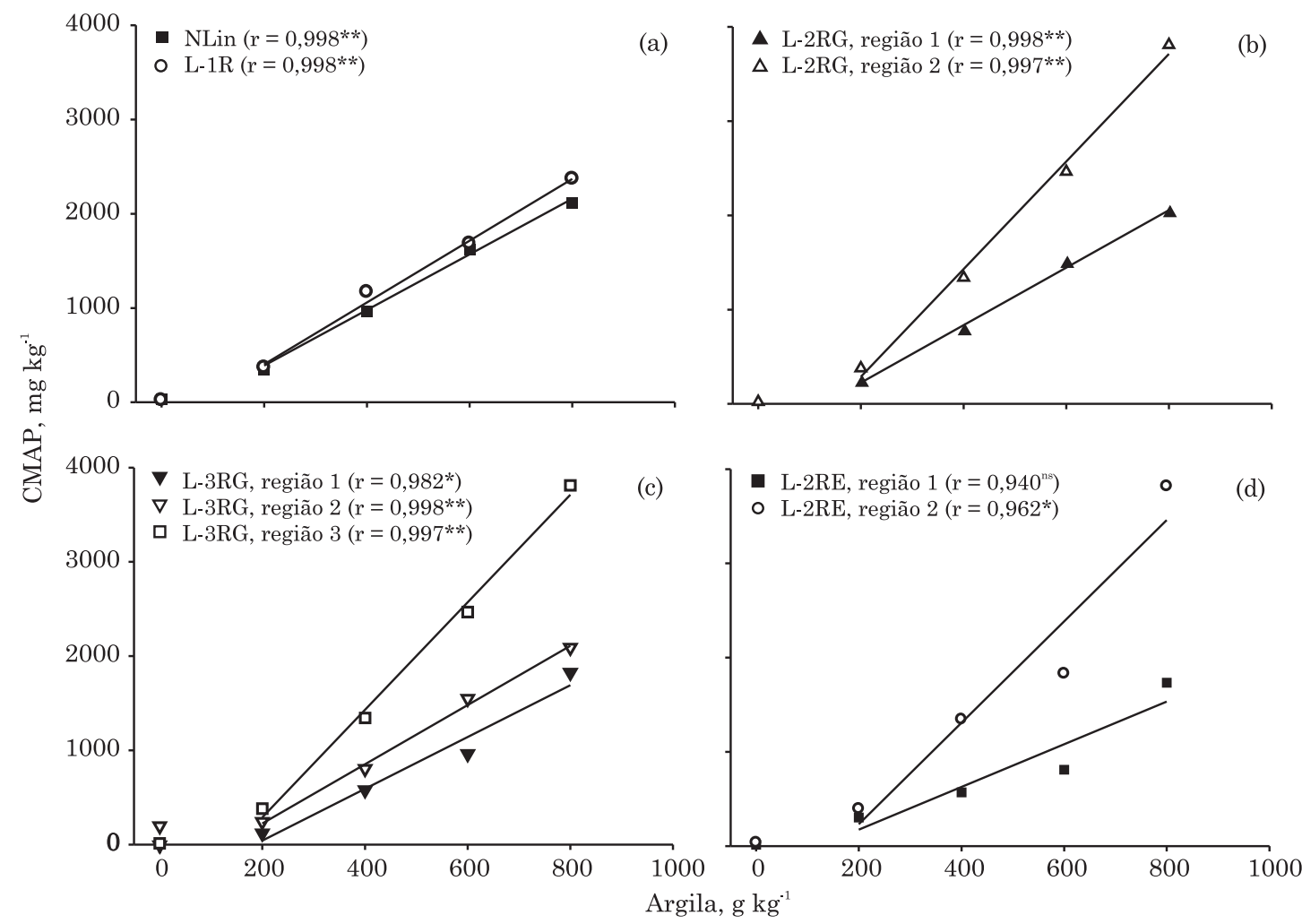

Figura 3. Relação entre o teor de argila do solo e a capacidade máxima de adsorção de fósforo (CMAP) em solos com diferentes teores de argila. Modos de ajuste do modelo: NLin = não linear com região única e L-1R = linearização com região única (a); L-2RG = linearização com duas regiões, ajuste gráfico (b); L-3RG = linearização com três regiões, ajuste gráfico (c); e L-2RE = linerização com duas regiões, ajuste estatístico (d). Por ser uma amostra composta apenas por areia, o tratamento " $0 \mathrm{~g} \mathrm{~kg}$-1 argila" foi excluido da análise de correlação. *: p<0,05; *** p<0,01; e ns: p>0,05. 


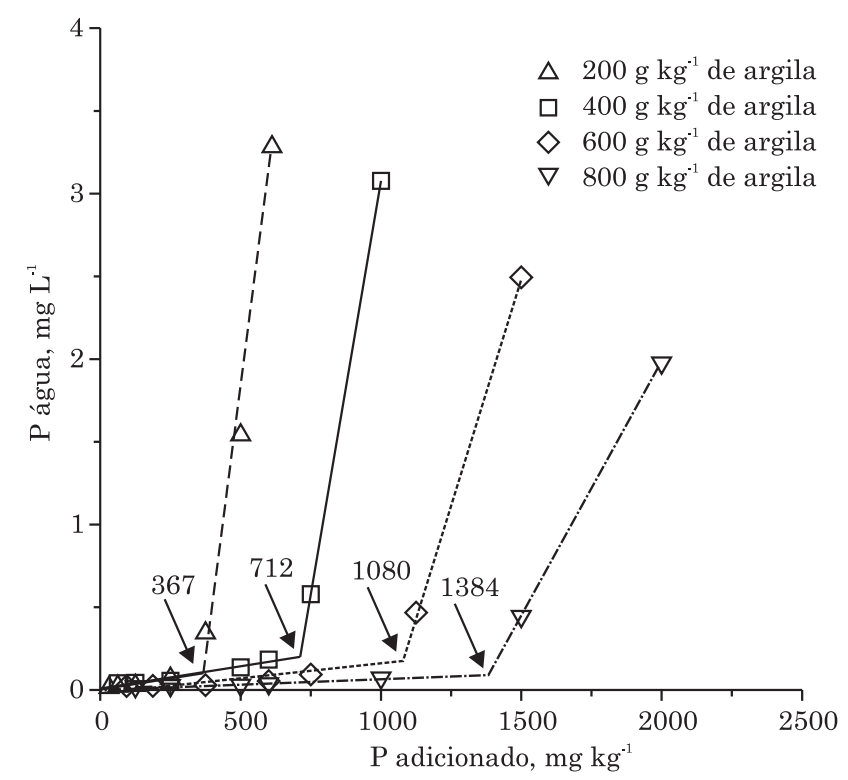

Figura 4. Teores de fósforo extraído com água após 20 dias de incubação de doses de fósforo em amostras com 200, 400, 600 e $800 \mathrm{~g} \mathrm{~kg}^{-1}$ de argila. Os valores indicados pelas setas representam a quantidade de fósforo adicionada em cada solo a partir da qual a taxa de liberação de fósforo para a água aumenta rapidamente.

II); 1,60 (L-3RG, região I); 1,80 (L-3RG, região II); 3,33 (L-3RG, região III); 1,31 (L-2RE, região I); e 3,11 (L-2RE, região II). Esses resultados demonstraram que o coeficiente angular foi mais elevado $(3,11$ a 3,33) quando se usou a CMAP da última região dos modelos das multirregiões. Assim, provavelmente a CMAP da última região seria mais adequada para estimar a capacidade de adsorção de $\mathrm{P}$ em longo prazo, como argumentado por Alvarez V. \& Fonseca (1990); no entanto, para isso, seriam necessários estudos complementares para testar essa hipótese.

Pelo exposto anteriormente, ficou evidente que os valores de $\mathrm{k}$ não foram coerentes quando utilizado o ajuste não linear. Quando usados modelos linearizados, os valores de $\mathrm{k}$ foram coerentes com as características dos solos utilizados, mas, no caso de linearização com regiões múltiplas, apenas o $\mathrm{k}$ calculado para a primeira região de adsorção apresentou-se coerente. Para os modelos linearizados com múltiplas regiões, embora os valores de CMAP tenham sido coerentes, os valores estimados para a última região foram sempre muito superiores aos valores encontrados por meio da incubação do solo com doses de P por 20 dias. Isso sugeriu que quando usados modelos linearizados com uma ou duas regiões, os valores de CMAP e k devem ser obtidos da primeira região. Quando utilizados modelos com três regiões, o valor de $\mathrm{k}$ deve ser obtido da primeira região, e o de CMAP, da segunda. Contudo, alerta-se que os modelos linearizados não possuem completa independência entre os fatores, diminuindo a variabilidade e aumentando artificialmente os coeficientes de determinação das equações ajustadas (Harter, 1984; Bolster \& Hornberger, 2007; Barrow, 2008).

\section{CONCLUSÕES}

1. Todos os métodos de ajuste utilizados estimaram valores de CMAP proporcionais ao teor de argila dos solos; porém, nos ajustes linearizados de regiões múltiplas, os valores da CMAP estimados para a última região foram inadequados quando comparados com os de adsorção obtidos pela incubação do solo com $\mathrm{P}$ por 20 dias.

2. Os valores da constante de energia de ligação foram coerentemente estimados pelo modelo linearizado na região única e pela primeira região dos modelos linearizados de regiões múltiplas, enquanto o ajuste não linear não estimou adequadamente os valores da constante de energia de ligação.

\section{LITERATURA CITADA}

ALMEIDA, J.A.; TORRENT, J. \& BARRÓN, V. Cor de solo, formas do fósforo e adsorção de fosfatos em Latossolos desenvolvidos de basalto do extremo-sul do Brasil. R. Bras. Ci. Solo, 27:985-1002, 2003.

ALVAREZ V., V.H. \& FONSECA, D.M. Definição de doses de fósforo para determinação da capacidade máxima de adsorção de fosfatos e para ensaios de casa de vegetação. R. Bras. Ci. Solo, 14:49-55, 1990.

BARROW, N.J. The description of sorption curves. Eur. J. Soil Sci., 59:900-910, 2008.

BOLSTER, C.H. \& HORNBERGE, G.M. On the use of linearized Langmuir equations. Soil Sci. Soc. Am. J., 71:1796-1806, 2007.

CASAROLI, D. \& van LIER, Q.J. Critérios para determinação da capacidade de campo. R. Bras. Ci. Solo, 32:59-66, 2008.

COMISSÃO DE QUÍMICA E FERTILIDADE DO SOLO CQFSRS/SC. Manual de adubação e de calagem para os Estados do Rio Grande do Sul e Santa Catarina. 10.ed. Porto Alegre, 2004. 400p.

CORRÊA, M.R.; NASCIMENTO, C.W.A. \& ROCHA, A.T. Adsorção de fósforo em dez solos do Estado de Pernambuco e suas relações com parâmetros físicos e químicos. Acta Sci. Agron., 33:153-159, 2011.

EMPRESA BRASILEIRA DE PESQUISA AGROPECUÁRIA EMBRAPA. Manual de métodos de análises de solo. 2.ed. Rio de Janeiro, Embrapa - CNPS, 1997. 212p.

FALCÃO, N.P.S. \& SILVA, J.R.A. Características de adsorção de fósforo em alguns solos da Amazônia Central. Acta Amaz., 34:337-342, 2004. 
FARIAS, D.R.; OLIVEIRA, F.H.T.; SANTOS, D.; ARRUDA, J.A.; HOFFMANN, R.B. \& NOVAIS, R.F. Fósforo em solos representativos do Estado da Paraíba. I - Isotermas de adsorção e medidas do fator capacidade de fósforo. R. Bras. Ci. Solo, 33:623-632, 2009.

HARTER, R.D. Curve-fit errors in Langmuir adsorption maxima. Soil Sci. Soc. Am. J., 48:749-752, 1984.

HUSSAIN, A.; GHAFOOR, A. \& MURTAZA, G. Use of models for phosphorus adsorption on some sodic soils of Punjab. Intern. J. Agric. Biol., 8:241-248, 2006.

MULJADI, D.; POSNER, A.M. \& QUIRK, J.P. The mechanism of phosphate adsorption by kaolinite, gibbsite, and pseudoboehrnite. Part I. The isotherms and the effect of $\mathrm{pH}$ on adsorption. J. Soil Sci., 17:222229,1966 .

MULJADI, D.; POSNER, A.M. \& QUIRK, J.P. The mechanism of phosphate adsorption by kaolinite, gibbsite, and pseudoboehrnite. Part II. The location of the adsorption sites. J. Soil Sci., 17:230-237, 1966b.

MULJADI, D.; POSNER, A.M. \& QUIRK, J.P. The mechanism of phosphate adsorption by kaolinite, gibbsite, and pseudoboehrnite. Part III. The effect of temperature on adsorption. J. Soil Sci., 17:238-247, 1966c.

MURPHY, J. \& RILEY, J.P. A modified single solution method for the determination of phosphate in natural waters. Anal. Chim. Acta, 27:31-36, 1962.
NOVAIS, R.F. \& SMYTH, T.J. Fósforo em solo e planta em condições tropicais. Viçosa, MG, Universidade Federal de Viçosa, 1999. 399p.

PARFITT, R.L. Anion adsorption by soils and soil materials. Adv. Agron., 30:1-46, 1978.

RANNO, S.K.; SILVA, L.S.; GATIBONI, L.C. \& RHODEN, A.C. Capacidade de adsorção de fósforo em solos de várzea do Estado do Rio Grande do Sul. R. Bras. Ci. Solo, 31:21-28, 2007.

R DEVELOPMENT CORE TEAM. R: A language and environment for statistical computing. Vienna, R Foundation for Statistical Computing, 2012. ISBN 3900051-07-0, URL <http://www.R-project.org>

RHEINHEIMER, D.S.; ANGHINONI, I. \& CONTE, E. Sorção de fósforo em função do teor inicial e de sistemas de manejo de solos. R. Bras. Ci. Solo, 27:41-49, 2003.

SAS Institute Inc® SAS Ver. 9.1. 3 Cary, NC, USA. Lic. UDESC, 2003.

TEDESCO, M.J.; GIANELLO, C.; BISSANI, C.A.; BOHNEN, H. \& VOLKWEISS, S.J. Análise de solo, plantas e outros materiais. Porto Alegre, UFRGS, 1995. 174p.

VALlADARES, G.S.; PEREIRA, M.G. \& ANJOS, L.H.C. Adsorção de fósforo em solos de argila de atividade baixa. Bragantia, 62:111-118, 2003.

WANG, Q. \& LI, Y. Phosphorus adsorption and desorption behavior on sediments of different origins. J. Soils Sed., 10:1159-1173, 2010. 